\title{
Abbau der kalten Progression seit 2016 - eine Zwischenbilanz
}

\author{
Seit 2016 findet ein Abbau der kalten Progression statt. Der Autor zeigt beispielhaft, inwiefern \\ die Steuerzahler:innen durch diese Reform in den vergangenen fünf Jahren entlastet wurden \\ und wertet dies als Zwischenerfolg für den Bund der Steuerzahler. Dieser Fortschritt sollte \\ jedoch durch weitere Reformen abgesichert werden.
}

Die kalte Progression - ein „ganz legaler Steuertrick“ der Politik - hat Jahrzehnte für ungerechtfertigte Steuermehreinnahmen gesorgt. Das Prinzip war einfach. Auch Einkommen, die zwar nominal, aber nicht real stiegen, führten zu höheren Durchschnittssteuersätzen. Dadurch wuchs die Steuerlast, obwohl die wirtschaftliche Leistungsfähigkeit der Steuerzahler:innen gleich geblieben war. Dieser finanzwissenschaftlich problematische Umstand ist von Seiten der politisch Verantwortlichen lange Zeit ignoriert, verneint oder kleingeredet worden. 2015 gelang der politische Durchbruch. Beschlossen wurde, fortan die kalte Progression im Einkommensteuertarif endlich zu Gunsten der Steuerzahlenden zu berücksichtigen. Das ist dann auch in allen Einkommensteuertarifen von 2016 bis 2021 geschehen.

Technisch erfolgt der Abbau der kalten Progression so, dass die Tarifeckwerte um die Inflationsrate erhöht werden. Dadurch bleiben die Durchschnittssteuersätze für real konstante Einkommen ebenfalls konstant. Für real gestiegene Einkommen wachsen die Durchschnittssteuersätze zwar weiterhin; aber weniger stark als früher, da nunmehr das lediglich inflationsbedingte „Pseudowachstum“ der Leistungsfähigkeit unberücksichtigt bleibt. Das war und ist ein wichtiger Zwischenerfolg für den Bund der Steuerzahler und alle anderen, die lange für den Abbau der kalten Progression geworben haben. Eine Zwischenbilanz nach fünf Reformjahren zeigt, dass die Reformeffekte trotz niedriger Inflationsraten durchaus beachtlich sind.

\section{Tarifentwicklung seit 2010}

Die Grundfreibeträge in den Tarifen 2010 bis 2021 stiegen in Anlehnung an den jeweiligen Existenzminimumbericht der Bundesregierung. Die übrigen Tarifeckwerte blieben

(C) Der/die Autor:in(nen) 2021. Open Access: Dieser Artikel wird unter der Creative Commons Namensnennung 4.0 International Lizenz veröffentlicht (creativecommons.org/licenses/by/4.0/deed.de).

Open Access wird durch die ZBW - Leibniz-Informationszentrum Wirtschaft gefördert. von 2010, dem Jahr der letzten großen Tarifreform, bis 2015 unverändert. Somit war in jenen Jahren ein großer Reformstau bezüglich der kalten Progression entstanden.

In den Einkommensteuertarifen 2016 bis 2021 hat dann die Gesetzgebung zur Dämpfung der kalten Progression die Tarifeckwerte entsprechend der Verbraucherpreisentwicklung angehoben (vgl. Tabelle 1). Als Resultat sind diese Eckwerte im aktuellen Einkommensteuertarif 2021 um 9,5\% größer als 2015. Diese Erhöhungen der Eckwerte bedeuten grafisch betrachtet eine Rechtsverschiebung der Grenzsteuerkurve (vgl. Abbildung 1) und damit eine Abflachung der Durchschnittssteuerkurve (vgl. Abbildung 2).

\section{Reformeffekte seit 2015}

Flachere Durchschnittssteuerkurven, also kleinere Durchschnittssteuersätze, führen zu geringeren Steuerlasten für gegebene Einkommen. Die Steuerlastsenkungen 2021 gegenüber dem Tarif 2015 resultieren allerdings aus zwei unterschiedlichen Komponenten. Die erste Komponente rührt aus den Anhebungen der Grundfreibeträge, die von 2015 bis 2021 aus verfassungsrechtlichen Gründen ohnehin nötig gewesen wären. Die zweite Komponente beinhaltet den zusätzlichen Entlastungseffekt infolge der Inflationierung der Eckwerte, also durch den Abbau der kalten Progression. Das ist die eigentliche Reformdividende, welche die Steuerzahlenden seit 2016 genießen können.

Zur Bezifferung dieser Reformdividende haben wir fiktive Einkommensteuertarife kalkuliert, die sich ergeben hätten, wenn ab 2016 (wie zuvor) lediglich die Grundfreibeträge

Matthias Warneke ist wissenschaftlicher Leiter des Deutschen Steuerzahlerinstituts des Bundes der Steuerzahler e. V., Berlin. 
Tabelle 1

Eckwerte der Einkommensteuertarife seit 2010

\begin{tabular}{lcccc}
$\begin{array}{l}\text { Einkommen- } \\
\text { steuertarife }\end{array}$ & 2010 & 2015 & 2021 & $\begin{array}{c}\text { Anstieg der Eck- } \\
\text { werte seit 2015 }\end{array}$ \\
\hline $\begin{array}{l}\text { Grundfreibe- } \\
\text { trag }\end{array}$ & $8.004 €$ & $8.472 €$ & $9.744 €$ & $\begin{array}{l}15 \% \text { (gemäß ver- } \\
\text { fassungsrechtlicher } \\
\text { Vorgaben) }\end{array}$ \\
\hline 1. Eckwert & $13.470 €$ & $14.754 €$ & $9,5 \%$ \\
\hline 2. Eckwert & $52.882 €$ & $57.919 €$ & $9,5 \%$ \\
\hline 3. Eckwert & $250.731 €$ & $274.613 €$ & $9,5 \%$
\end{tabular}

Quelle: jeweils geltende Einkommensteuergesetze und eigene Berechnungen.

erhöht, die sonstigen Eckwerte aber nicht an die Inflation angepasst worden wären (vgl. Tabelle 1). So enthält beispielsweise der hier verwendete fiktive Tarif 2021 den gleichen Grundfreibetrag wie im geltenden Tarif 2021, im Übrigen aber die Eckwerte des Einkommensteuertarifs 2015.

Grafische Vergleiche für 2021 zeigen exemplarisch die Unterschiede zwischen solch einem fiktiven Tarif und dem geltenden Tarif bezüglich der Grenzsteuersätze (vgl. Abbildung 3) und der Durchschnittssteuersätze (vgl. Abbildung 4). Auf Basis der fiktiven Einkommensteuertarife lässt sich aber nicht nur grafisch, sondern auch numerisch zeigen, welche Entlastungen die Steuerzahlenden durch den Abbau der kalten Progression effektiv erfahren haben. Für 2021 zeigt Tabelle 2 einige Beispiele: Ein Single mit einem zu versteuernden Jahreseinkommen von 40.000 Euro würde in 2021343 Euro mehr Einkommensteuer zahlen, wenn die kalte Progression nicht seit 2016 abgebaut worden wäre. Zudem belegen diese Beispiele einen bekannten Effekt. Die relative Steuerentlastung, also die Ersparnis gemessen an der individuellen Steuerlast, ist umso größer, je kleiner das Einkommen ist. Bezieher:innen kleiner und mittlerer Einkommen profitieren also vom Abbau der kalten Progression in besonderer Weise.

Wie groß dieser Reformeffekt 2021 nun für alle Steuerzahlenden insgesamt ist, lässt sich auf Basis des fiktiven Einkommensteuertarifs ebenfalls abschätzen. Nach unseren Hochrechnungen wäre die Steuerzahlerlast 2021 rund 9,6 Mrd. Euro höher, wenn der erwähnte fiktive Tarif 2021 gelten würde. Ein analoger Vergleich der jeweils geltenden Tarife der Vorjahre mit den dazugehörigen fiktiven Tarifen ermöglicht es, auch für den gesamten Reformzeitraum 2016 bis 2021 abzuschätzen, wie groß die Entlastungseffekte insgesamt (Einkommensteuer und Solidaritätszuschlag) waren (vgl. Tabelle 3). Enthalten sind nachrichtlich zudem die Indexierungsraten, welche die Gesetzgebung jeweils entsprechend der von ihr berücksichtigten Verbraucherpreisdaten zur Tarifbestimmung herangezogen hat.
Abbildung 1

Grenzsteuersätze in den Tarifen 2015 und 2021

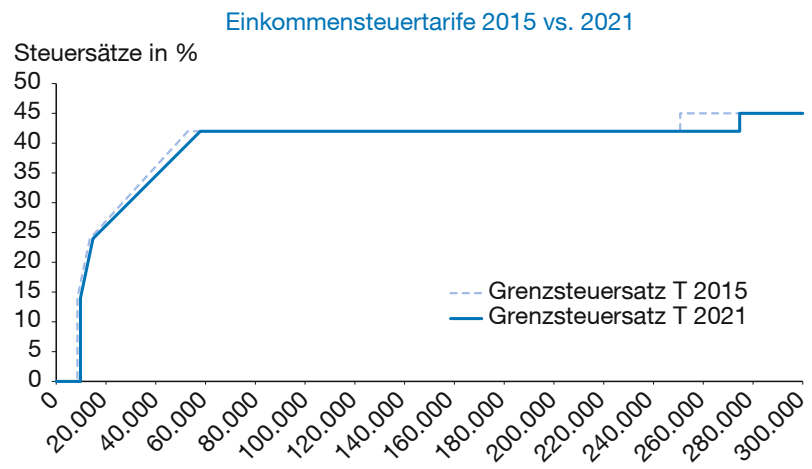

zu versteuerndes Jahreseinkommen in Euro

Quelle: eigene Berechnungen.

\section{Abbildung 2}

Durchschnittssteuersätze in den Tarifen 2015 und 2021

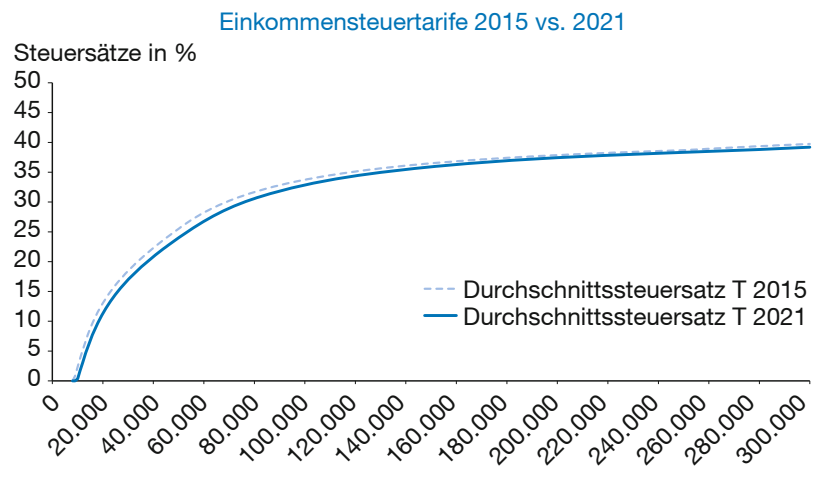

zu versteuerndes Jahreseinkommen in Euro

Quelle: eigene Berechnungen.

\section{Abbildung 3}

Exemplarische Reformeffekte 2021 im Grenzsteuerbereich

Einkommensteuertarife 2021 mit und ohne Reform

Steuersätze in \%

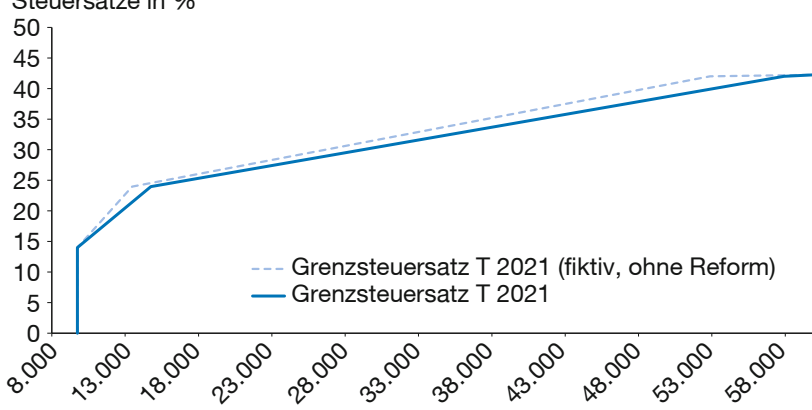

zu versteuerndes Jahreseinkommen in Euro

Quelle: eigene Berechnungen. 
Abbildung 4

Exemplarische Reformeffekte 2021 im

Durchschnittssteuerbereich

Einkommensteuertarife 2021 mit und ohne Reform

Steuersätze in \%

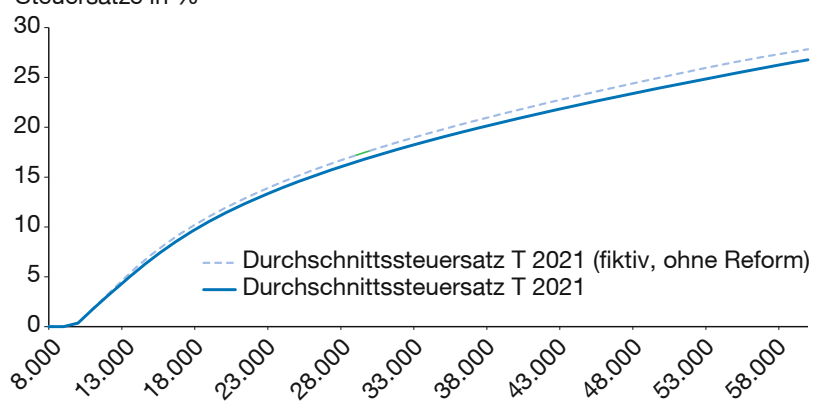

zu versteuerndes Jahreseinkommen in Euro

Quelle: eigene Berechnungen.

Tabelle 2

Exemplarische Reformeffekte 2021 für ausgewählte Steuerzahler:innen

Entlastung am Beispiel eines Singles ohne Kinder (in Euro)

\begin{tabular}{|c|c|c|c|c|}
\hline \multirow{3}{*}{$\begin{array}{l}\text { zu ver- } \\
\text { steuerndes } \\
\text { Jahres- } \\
\text { einkommen }\end{array}$} & \multicolumn{2}{|c|}{$\begin{array}{l}\text { Einkommensteuerlast } \\
2021 \mathrm{im}\end{array}$} & \multirow{2}{*}{\multicolumn{2}{|c|}{$\begin{array}{l}\text { Betrag, um den die Steuerlast } \\
2021 \text { höher wäre, wenn die } \\
\text { kalte Progression } \\
\text { nicht seit } 2016 \text { abgebaut } \\
\text { worden wäre }\end{array}$}} \\
\hline & \multirow[t]{2}{*}{$\begin{array}{l}\text { fiktiven } \\
\text { Tarif }(A)\end{array}$} & \multirow[t]{2}{*}{$\begin{array}{l}\text { geltenden } \\
\text { Tarif (B) }\end{array}$} & & \\
\hline & & & $\begin{array}{l}\text { absolut } \\
\text { (A-B) }\end{array}$ & $\begin{array}{c}\text { relativ } \\
(\mathrm{A}-\mathrm{B}) / \mathrm{B} \text { (in \%) }\end{array}$ \\
\hline 20.000 & 2.370 & 2.266 & 104 & 4,6 \\
\hline 40.000 & 8.676 & 8.333 & 343 & 4,1 \\
\hline 80.000 & 25.097 & 24.463 & 634 & 2,6 \\
\hline
\end{tabular}

Quelle: eigene Berechnungen.

\section{Fazit und Ausblick}

Gemäß unserer Schätzung beträgt die Entlastung der Steuerzahler:innen durch den Abbau der kalten Progression von 2016 bis 2021 voraussichtlich fast 32 Mrd. Euro. Dabei handelt es sich jedoch keineswegs um eine politische Großzügigkeit. Der Abbau der kalten Progression war und ist finanzwissenschaftlich geboten, was inzwischen auch anerkannt wird. Die Reformen hätten daher schon viele Jahre früher ansetzen müssen. Der Abbau der
Tabelle 3

Abbau der kalten Progression - effektive Entlastungseffekte

\begin{tabular}{lcc} 
Jahr & $\begin{array}{c}\text { Indexierungsrate } \\
\text { (in \%) }\end{array}$ & $\begin{array}{c}\text { Effektive Gesamtentlastung der } \\
\text { Steuerzahler:innen durch den Abbau } \\
\text { der kalten Progression (in Mrd. Euro) }\end{array}$ \\
\hline 2016 & 1,48 & 1,4 \\
\hline 2017 & 0,73 & 2,2 \\
\hline 2018 & 1,65 & 4,1 \\
\hline 2019 & 1,84 & 6,0 \\
\hline 2020 & 1,95 & 8,2 \\
\hline 2021 & 1,52 & 9,6 \\
\hline $2016 / 2021$ & 9,52 & 31,6 \\
\hline
\end{tabular}

Quelle: jeweilige Gesetzentwürfe, eigene Berechnungen auf Basis des Statistischen Bundesamts (2016).

kalten Progression seit 2016 ist in jedem Fall ein großer Fortschritt. Er muss jedoch weiter abgesichert werden, indem - wie von uns seit Langem vorgeschlagen und in einem Gesetzentwurf konkretisiert - eine quasiautomatische Tarifindexierung durch einen „Tarif auf Rädern“ erfolgt. In einem weiteren Schritt muss zudem die kalte Progression im weiteren Sinne („heimliche Steuererhöhungen") abgebaut werden. Hierzu ist eine Tarifindexierung auf Basis der durchschnittlichen Nominallohnerhöhung nötig. Denn eine durchschnittliche Nominallohnerhöhung bedeutet eine im Vergleich zu anderen Einkommensteuerzahlenden unveränderte Leistungsfähigkeit, was insofern auch keine steigenden Durchschnittssteuersätze rechtfertigt. Auch hier ist mit politischen Widerständen zu rechnen. Der inzwischen erreichte Abbau der kalten Progression hat jedoch gezeigt, dass sich Beharrlichkeit in politischen Debatten auszahlt. Daher wird der Bund der Steuerzahler an der Forderung nach einem „Tarif auf Rädern“ sowie einem Abbau der „,heimlichen Steuererhöhungen" festhalten.

\section{Literatur}

Statistisches Bundesamt (2016), Lohn- und Einkommenssteuerstatistik, Fachserie 14, Reihe 7.1

Deutsches Steuerzahlerinstitut des Bundes der Steuerzahler (2014), Abbau der kalten Progression - Teil einer Steuerbremse, DSi Schrift Nr. 2.

Title: Curbing the Fiscal Drag - A Provisional Assessment

Abstract: It took decades of political discussion for lawmakers to begin adjusting the income tax tariff for inflation in order to curb the fiscal drag. Since 2016, the fiscal drag has been reduced by almost $€ 32$ billion according to our estimates. That is certainly a substantial amount given the low inflation rates in the period 2016-2021. We illustrate these effects graphically and also numerically for individual taxpayers. We find that further reforms are needed. A specific formula should be integrated into the Income Tax Act so that the tariff is adjusted automatically to inflation. In the long run, average income growth rates should be used for indexation instead of inflation rates. JEL Classification: $\mathrm{H} 2 \mathrm{O}, \mathrm{H} 24$ 